\section{A155 ASSOCIATION BETWEEN CLINICAL RESPONSE AND CIRCULATING B CELL SUBSETS DURING B CELL DEPLETION THERAPY FOR SYSTEMIC LUPUS ERYTHEMATOSUS}

E M Vital, ${ }^{1,2}$ S Dass, ${ }^{1,2}$ M H Buch, ${ }^{1,2}$ K Henshaw, ${ }^{1,2}$ A C Rawstron, ${ }^{3}$ F Ponchel, P Emery ${ }^{1}$ NIHR Leeds Musculoskeletal Biomedical Research Unit, Leeds Teaching Hospitals NHS Trust, Leeds, UK; ${ }^{2}$ Section of Musculoskeletal Disease, Leeds Institute of Molecular Medicine, University of Leeds, Leeds, UK; ${ }^{3}$ Haematological Malignancy Diagnostic Service, Leeds Teaching Hospitals NHS Trust, Leeds, UK

10.1136/ard.2010.149005.22

Background B cell depletion using rituximab appears effective in systemic lupus erythematosus (SLE) in open label case series, but initial response, effect on autoantibody titres and time to relapse are highly variable.

Objective To investigate relationships between clinical response, B cell subsets and autoantibody titres during rituximab therapy for SLE.

Materials and methods 41 patients with active SLE received a standard regime of rituximab with intravenous and oral steroids. Clinical response and relapse were defined using BILAG with criteria for response, non-response and relapse. Naïve(CD19+CD27-CD38+/-), memory(CD19+CD27+CD38+/-) and plasmablast(CD19+CD27++CD38++) numbers were measured by flow cytometry evaluating 500000 events at baseline, after each infusion of rituximab and then three monthly. BILAG and $B$ cell numbers were compared between patient groups using non-parametric tests and anti-dsDNA and immunoglobulin titres were log transformed and compared using t tests.

Results There was significant reduction in global BILAG $(p<0.0001)$ and $82 \%$ of patients responded at 6 months. Time to relapse was highly variable. $50 \%$ of patients relapsed after 6-18 months (earlier relapse); the remainder relapsed at a slower rate (later relapse). B cell depletion and repopulation were variable and predictive of these clinical outcomes. 21 patients had persistent B cells after both infusions of rituximab, which included all 7 patients with non-clinical response $(p=0.012)$. Memory B cell $(p=0.02)$ and plasmablast $(p<0.001)$ repopulation after 6 months was markedly greater in patients with earlier relapse. No differences were identified between dsDNA, ENA or IgM, IgA and IgG titres at baseline or 6 months comparing patients with response/non-response or earlier/later relapse. Patients with antibodies to extractable nuclear antigens (Ro, La, Sm, RNP) at baseline had higher plasmablast numbers at baseline $(p=0.025)$. Patients with persistent B cells after both infusions of rituximab had significantly higher levels of antidsDNA at 6 months $(p=0.012)$. Notably, several patients had clinical response lasting several years with sustained suppression of memory B cell and plasmablast numbers to very low levels but continued high anti-dsDNA titres.

Conclusions In this study the strongest relationship was between initial degree of $B$ cell depletion and subsequent clinical response, and between memory $B$ and plasmablast repopulation and subsequent relapse. In this series, reduction of autoantibody titre was greater when plasmablast numbers were undetectable 
after rituximab, but normalisation of autoantibody titre did not appear to be required for good clinical response. 